\title{
PIXEL, Plataforma para Integração de Experimentos de Interoperabilidade em Sistemas Legados de Saúde Pública
}

\author{
Leonardo Nascimento ${ }^{1}$, Paulo Castro ${ }^{2}$, Mauro Oliveira ${ }^{1}$, Fabio Jose ${ }^{1}$, Valter \\ Costa $^{1}$, Cesar Moura ${ }^{1}$, Renato Freitas ${ }^{1}$, Odorico Monteiro $^{3}$ \\ ${ }^{1}$ Instituto Federal de Educação, Ciência e Tecnologia do Ceará \\ Ceará - Brasil \\ ${ }^{2}$ Universidade Estadual do Ceará \\ Ceará - Brasil \\ ${ }^{3}$ Fundação Oswaldo Cruz - Fiocruz Ceará \\ Ceará - Brasil

\begin{abstract}
\{leonardo.gomes, renato.freitas\} @ppgcc.ifce.edu.br, paullocasttro@hotmail.com, \{amauroboliveira, prof.fabiojose, odorico0811\}@gmail.com, \{valter.costa, cesarolavo\}@ifce.edu.br
\end{abstract}

\begin{abstract}
Health information systems (SIS) are increasing in complexity and volume of data. The data does not always follow a pattern, aggravating the integration of services, especially when it involves legacy systems. This work proposes PIXEL, a platform to integrate interoperability experiments in legacy public health systems. This solution was applied to GISSA, an intelligent system in the health area that uses machine learning to generate alerts and support the decision making of its users. PIXEL performed the systems integration and implements the initial three levels of interoperability, as proposed by the Society for Health Information and Management Systems (HIMSS).
\end{abstract}

Resumo. Sistemas de informações em saúde (SIS) vêm aumentando em complexidade e volume de dados. Os dados nem sempre seguem um padrão, agravando a integração de serviços, especialmente quando envolve sistemas legados. Este trabalho propõe a PIXEL, uma plataforma para integrar experimentos de interoperabilidade em sistemas legados de saúde pública. Essa solução foi aplicada ao GISSA, um sistema inteligente na área da saúde que utiliza aprendizagem de máquina para gerar alertas e apoiar à tomada de decisão de seus usuários. A PIXEL realizou a integração de sistemas e implementa os três níveis iniciais de interoperabilidade, conforme proposto pela Society for Health Information and Management Systems (HIMSS).

\section{Introdução}

A grande diversidade dos dados e informações fornecidas à administração pública traz grandes desafios para cooperação e a formalização de processos, entre os quais a integração das fontes de informação das instâncias governamentais e privadas. De acordo com [Henning 2013], Governos falham em implementar interoperabilidade em seus sistemas públicos de saúde por diversas razões, principalmente por não cumprirem normas necessárias por parte dos componentes de uma rede de colaboração, o que impacta diretamente nos diferentes setores governamentais que têm a necessidade de integrar informações aos seus sistemas ou portais. Para [Mahmoud 2013], a necessidade de integrar sistemas não é um problema que deve ter uma solução única em determinado momento. É, antes, uma obrigação que os governos e organizações deverão satisfazer no futuro para os setores que utilizam tecnologias 
No setor de saúde, as entidades geram diariamente um denso volume de informações em formatos variados, provenientes de diferentes fontes de informação. Para agravar a situação, é comum que entidades adotem diferentes sistemas de informação para tratar e armazenar seus dados. Uma das prioridades destacadas por estas instituições está diretamente ligada à integração destes sistemas com os seus parceiros internos e externos, assegurando a troca de informação. A integração de SIS é uma característica fundamental do conceito interoperabilidade de sistemas, objeto deste trabalho. Assim, a complexidade da atividade na área de saúde exige SIS interoperáveis.

Para [dos Santos 2011], a interoperabilidade é a competência que sistemas têm de trocar, processar e compreender corretamente as informações, sendo requisito indispensável para a entrega flexível e a viabilização eletrônica de serviços governamentais. Na visão de [Vernadat 2007], o termo interoperabilidade está relacionado à capacidade de utilizar, por parte do processo ou sistema, as informações de outro sistema por meio de padrões estabelecidos.

Este trabalho apresenta a PIXEL, uma Plataforma para Integração de Experimentos de Interoperabilidade em Sistemas Legados de Saúde Pública. Esta solução foi implementada no GISSA [Oliveira et al. 2010], um sistema inteligente na área materno-infantil que usa machine learning e ontologias na geração de alertas e apoio à tomada de decisão de seus usuários. Assim, é apresentada a proposta da plataforma PIXEL, que trata os problemas de interoperabilidade no GISSA. A PIXEL foi, portanto, integrada ao GISSA possibilitando a agregação de novos serviços ao sistema legado. Além de resolver o problema de integração de serviços, demonstrado no Estudo de Caso, a proposta se apresenta como uma solução a ser adotada em outros sistemas de similar interesse e faz uso da GIRLS [Gomes et al. 2019] na sua estrutura, disponibilizando serviços de interoperabilidade para Registro Eletrônico de Saúde (RES) nos padrões Open Electronic Health Record (OpenEHR) e Fast Healthcare Interoperability Resources (FHIR).

Este trabalho está organizado da seguinte forma: A seção 2 discute os trabalhos relacionados à plataforma proposta. A seção 3 apresenta o GISSA, sistema integrado a PIXEL. A seção 4 descreve a solução PIXEL. A seção 5 descreve o Estudo de Caso, a aplicação da solução proposta no sistema de saúde GISSA. Por fim, na seção 6 são apresentadas as considerações finais sobre as soluções adotadas na PIXEL e a sua importância no contexto de integração e interoperabilidade de SIS.

\section{Trabalhos Relacionados}

No trabalho apresentado por [do Nascimento et al. 2013] é proposto a InteropFrame, uma ferramenta que sistematiza uma solução de interoperabilidade entre os sistemas baseados em estruturas distribuídas de modelos diferentes, permitindo que os componentes envolvidos na implementação de aplicações distribuídas conversem por intermédio de mecanismos automatizados de interoperabilidade. $\mathrm{O}$ trabalho feito por [Hidalgo-Delgado et al. 2019] apresenta uma ferramenta de interoperabilidade semântica baseada em dados para bibliotecas digitais. A ferramenta proposta é baseada em três camadas: suporte aos dados de aquisição, processo de publicação de dados vinculados e construção de serviços de valor agregado para as bibliotecas digitais comerciais. No trabalho realizado por [Amin et al. 2020], tem-se uma pesquisa que objetiva construir uma ferramenta para viabilizar a interoperabilidade entre sistemas de $e$-saúde. A interoperabilidade é alcançada por meio do paradigma de arquitetura orientada a serviços.

Observa-se com esses trabalhos a aderência da ideia de implementar ferramentas interoperáveis, porém diferentes dos trabalhos citados, a proposta de implementar uma plataforma que permita interoperabilidade entre sistemas de saúde através da especificação de modelos de comunicação. 


\section{GISSA, Governança Inteligente em Sistemas de Saúde}

O projeto GISSA é um sistema que surgiu a partir da plataforma LARIISA [Oliveira et al. 2010]. Trata-se de um sistema inteligente de governança para o apoio à tomada de decisão em ambientes de saúde, que teve seu foco inicial no projeto Rede Cegonha do Ministério da saúde, sendo apoiado pelo Financiamento de Estudos e Projetos. O GISSA é constituído por uma série de componentes que possibilitam a coleta, integração e visualização de informações relevantes aos procedimentos de tomada de decisão. A prova de conceito do GISSA foi realizada na cidade de Tauá - Ceará, tendo havido uma expansão do sistema para 20 municípios do estado do Ceará e de Pernambuco. A Figura 1 mostra a arquitetura do GISSA, seus componentes, conexões e fluxo de dados.

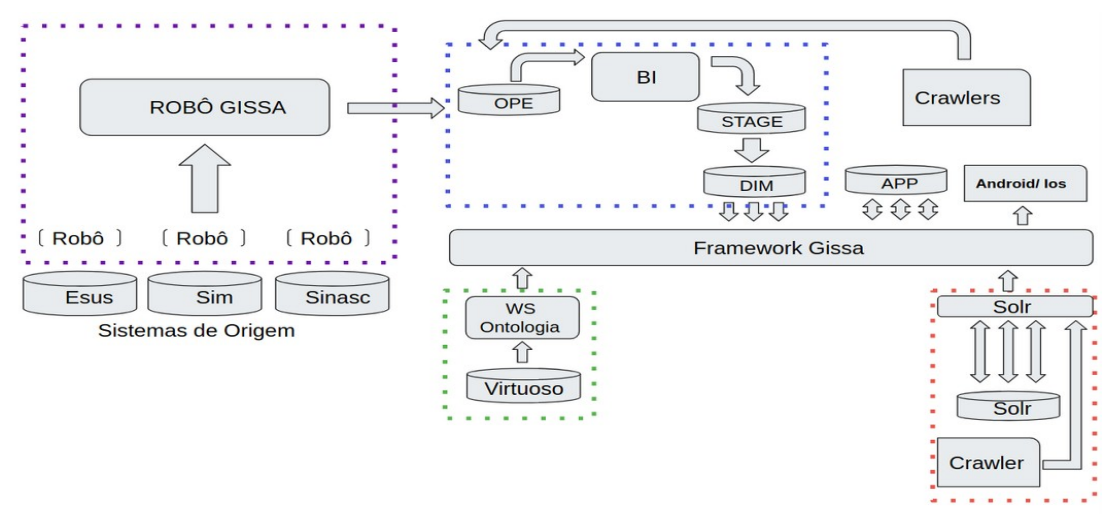

Figura 1. Arquitetura GISSA

O GISSA utiliza o Atlântico web framework (AWF), um framework proprietário que cria seus serviços fundamentais: Dashboard, indicadores, relatórios e alertas. As informações usadas no GISSA são oriundas de bases de dados tradicionais do DATASUS (ESUS, SIM, SINIASC, etc), obtidas por meio de autorização junto aos responsáveis de cada município. Para o povoamento das informações do GISSA, é necessária a execução da carga periódica por meio dos seus robôs de software instalados nas máquinas dos municípios que contêm as bases de dados.

Estas informações são gravadas no banco de dados Operacional (banco de dados com informações brutas e não tratadas) do GISSA que passa as informações para o componente de Business Inteligence (BI) e que usa Crawlers (mecanismo usando para facilitar insights importantes para as estratégias de colheita de informações). O componente de BI do GISSA cria as rotinas de execução das informações a serem refinadas nos bancos de dados Stage e Dimensional que tratam as informações de modo a deixá-las no formato final para serem exibidas nas telas do sistema. O fluxo do robô é realizado pelo Extract, Transform and Load (ETL) até o framework do GISSA. O framework do GISSA é assistido e supervisionado pelo banco de dados APP. Este banco de dados sumariza as informações do sistema e trata as questões de gestão e supervisão dos dados.

$\mathrm{O}$ framework do GISSA tem o potencial de agregar funcionalidades e serviços por meio da tecnologia REST em um estágio inicial para renderização (via $P O S T$ ). Atrelado ao AWF tem os recursos Web Semântica (WS), Solr e Android/Ios que serão explicados a seguir. O WS ontologia agrega valores de dados semanticamente ao banco de dados Virtuoso, o qual guarda informações com objetivo de realizar a inferência de dados. O Solr realiza a indexação de determinados dados promovendo a escalabilidade dos mesmos e os armazenando no banco de dados Solr, tendo como apoio um Crawler para capturar dados relevantes para otimização de alto volume de informações. Os recursos Android e Ios tratam-se das versões disponíveis para dispositivos móveis do GISSA. 
Diante desse contexto, pode-se notar que o GISSA é um sistema legado de informação na saúde, com adesão da tecnologia Rest no nível 0 e com um framework proprietário. Entretanto, ao tentar realizar a comunicação do GISSA com outro sistema que esteja além do escopo da operação dos robôs (ETL), tem-se acentuadas dificuldades de integração. Levando em consideração o cenário de difícil comunicação do GISSA, foi implementada a plataforma PIXEL, a qual cria serviços Restful que viabilizam a interoperabilidade de sistemas legados e faz utilização da GIRLS para usar os padrões OpenEHR e FHIR.

\section{Plataforma proposta PIXEL}

Este capítulo apresenta a PIXEL. Seguem em pormenores os pontos destacados nesta seção: cenário de aplicação, arquitetura e fluxo de funcionamento da PIXEL. De modo a constituir uma ferramenta que possibilita a criação de serviços a serem utilizados na integração de SIS.

\subsection{Cenário de Aplicação}

Considere-se, agora, o sistema GISSA como um componente em um contexto mais simplificado, ou seja, deseja-se integrar ao GISSA, um novo serviço externo ao escopo de operação dos robôs. Via de regra, todos os dados manipulados pelo AWF são provenientes da captura de informações de banco de dados governamentais (ESUS, SIM, SINASC, etc.), via robôs ETLs. Foram identificados aspectos das interoperabilidades fundamental, sintática e semântica.

Fundamental: Quanto ao aspecto de interoperabilidade comunicação, são identificados dois problemas a serem considerados neste cenário de aplicação:

1. Consumo de Informação: A comunicação do GISSA com qualquer sistema externo só pode ser feita via o POST, único verbo disponibilizado pelo back-end do GISSA e, portanto, usado pelo AWF.

2. Disponibilização de Informação: O GISSA não possui um mecanismo que disponibilize informações para uma comunicação com outro sistema ou componente em um mundo exterior ao seu.

Sintática: Uma vez resolvida a questão do consumo e disponibilidade da informação, o próximo passo é o tratamento sintático desta informação. No caso, tratarse-ia do mapeamento de informações (tamanho, tipos, nomes de campos, etc.) contidas em uma base de dados, transformando-as em um modelo de dados com a possibilidade de transformação/inserção em outra base.

Semântica: A plataforma PIXEL propõe a solução desses três aspectos de interoperabilidade, tendo sido implementados os aspectos de Comunicação (Consumo e Disponibilidade de Informação), Sintaxe (estrutura de banco de dados) e Semântica (OpenEHR e FHIR).

Para prover a interoperabilidade semântica em SIS que usam padrões de consensos como FHIR e OpenEHR, a PIXEL usa o GIRLS (Gateway para Interoperabilidade de Registro Eletrônico de Saúde em Sistema de Baixo Custo).

\subsection{Arquitetura da PIXEL}

A Figura 2 apresenta os principais componentes da arquitetura da plataforma PIXEL, a qual objetiva promover as interoperabilidades em diversos níveis entre SIS e integrar novos serviços em sistemas legados. São três os possíveis fluxos de informação entre as entidades comunicantes: (1) Entre sistemas legados, (2) Entre sistemas não legados e (3) Sistema legados e não legados. Considere-se, para melhor entendimento da arquitetura, um cenário em que a plataforma PIXEL está sendo utilizada entre dois sistemas legados, A e B. Neste cenário, objetiva-se mostrar no relatório do sistema $\mathrm{A}$, 
as informações coletadas de um banco de dados do sistema B. Para isto, serão realizados os seguintes passos:

Configuração: A iniciação da plataforma PIXEL requer as seguintes ações: (1) cadastrar os parâmetros de conexão para o servidor desejado do sistema B; (2) Estabelecer conexão com o servidor selecionado do sistema B; (3) Listar databases disponíveis do servidor selecionado do sistema B; (4) Selecionar database desejada do sistema B; (5) Listar tabelas disponíveis da database selecionada do sistema B; (6) Selecionar tabela do sistema B; (7) Gerar modelo de dados, tendo como parâmetro a tabela selecionada do sistema B; (8) Gerar o JAR.

Execução: Uma vez configurado, o JAR é gerado e publicado, permitindo que o sistema A possua uma API Restful intermediada pela PIXEL.

Operação: Esta etapa ficará a cargo do sistema A, consumindo as informações e interagindo com o sistema B. A seguir a Figura 2 apresenta a arquitetura da solução.

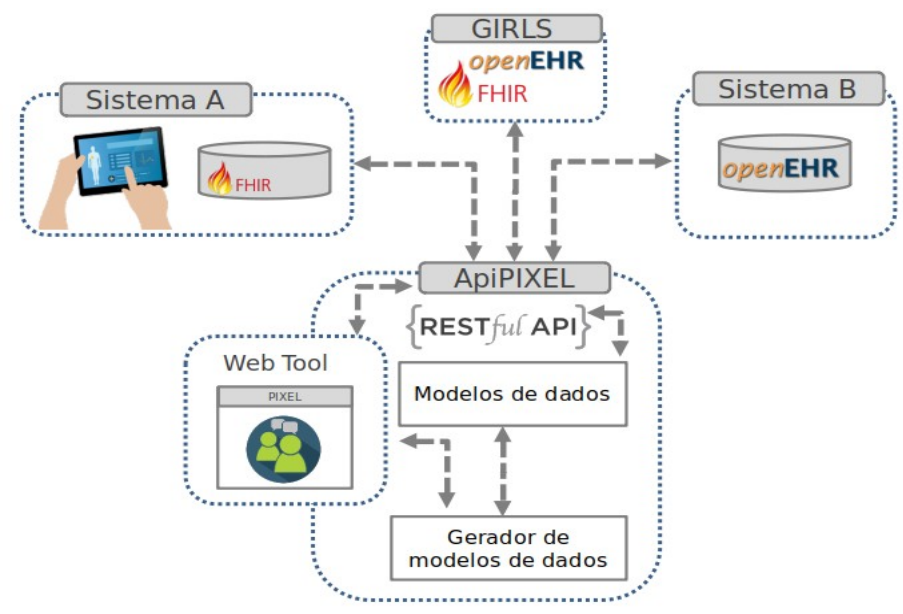

Figura 2. Arquitetura da solução proposta

Sistema A e Sistema B: Correspondem aos sistemas que vão conversar via PIXEL, sendo o Sistema A quem recebe as informações e o Sistemas B quem as envia.

GIRLS: Gateway usado para a conversão entre os padrões OpenEHR e FHIR, funcionando com uma estrutura da PIXEL para padronização das informações.

Web Tool: Interface gráfica da PIXEL onde é feita a escolha da base de dados e das tabelas usadas. As informações das tabelas são enviadas para o Gerador de modelos.

Gerador de modelos: Mecanismo que espelha a(s) tabela(s) selecionada(s) do sistema $\mathrm{B}$, realizando o(s) seu(s) mapeamento(s) e interligando os campos das informações desta(s) tabela(s) fornecendo as informações para o sistema A, possibilitando o consumo das informações da(s) tabela(s), com os campos já prédefinidos e prontos para a utilização gerando o modelos de dados.

Modelo de dados: Resultado do mapeamento da entidade do sistema B com suas características, propriedades e relacionamentos, possibilitando a operacionalidade no sistema A através de endpoints RestFul viabilizados pela PIXEL.

ApiPIXEL: É uma API RestFul orientada a modelos de dados que disponibiliza serviços, comportando-se como um gateway entre os dois sistemas comunicantes. A seguir as quatro principais funcionalidades da ApiPIXEL: (1) Importar o novo modelo criado pelo Gerador de Modelo; (2) Solicitar a API Restful para receber dados do sistema B usando o verbo apropriado; (3) Preencher o novo(s) modelo(s), estruturado no sistema A, pelo Gerador de Modelos; (4) Solicitar a API RestFul para enviar dados 
(recebidos do sistema B), já no novo modelo, para o Sistema de Consumo de Dados do sistema A, usando o verbo apropriado.

\subsection{Fluxo de funcionamento}

A Figura 3 apresenta o Business Process Model and Notation (BPMN) do fluxo de funcionamento da PIXEL e a seguir a sua explicação.

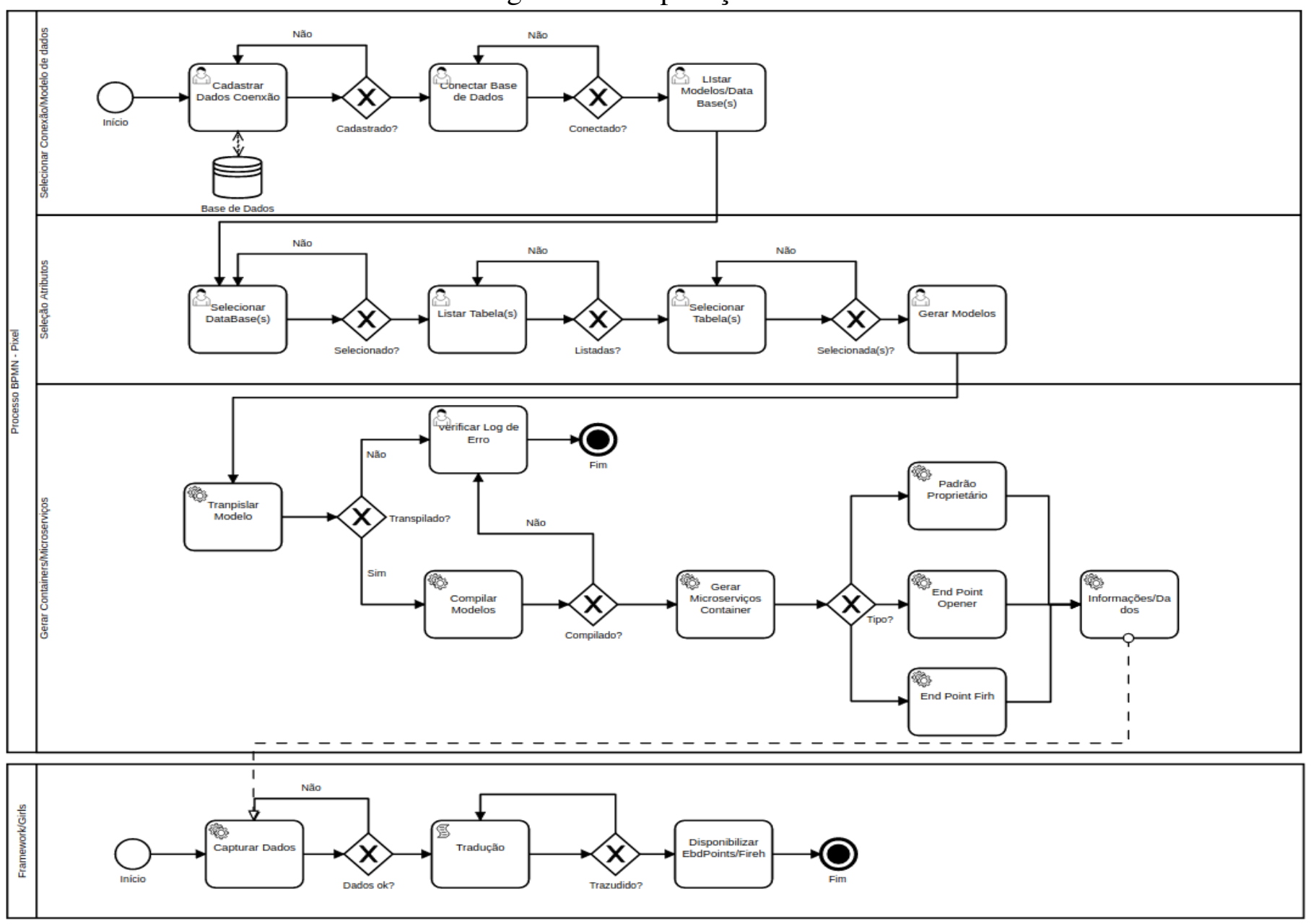

Figura 3. fluxo de funcionamento da arquitetura PIXEL em BPMN

1. Seleção de conexão: Neste momento é selecionado a base de dados e a tabela;

2. Seleção de atributos: Neste momento são filtradas as informações a serem convertidas em modelos de dados;

3. Geração de serviços: Neste momento são disponibilizados os serviços baseados (endpoints) nos modelos de dados escolhidos;

4. GIRLS: Neste momento as informações oriundas da PIXEL são enviadas para GIRLS. A GIRLS retornará as informações para a PIXEL nos padrões OpenEHR e FHIR.

\section{Estudo de Caso}

A plataforma PIXEL foi aplicada em duas simulações de municípios gerenciados pelo 
sistema GISSA, tendo cada simulação sido executada em máquinas diferentes, cada uma contendo sua base de dados local. Um dos municípios disponibiliza suas informações (via tecnologia REST) enquanto o outro município pode acessar, via interface web da plataforma proposta, essas informações. A Figura 4 apresenta a arquitetura do GISSA usando a PIXEL, configurando este estudo de caso.

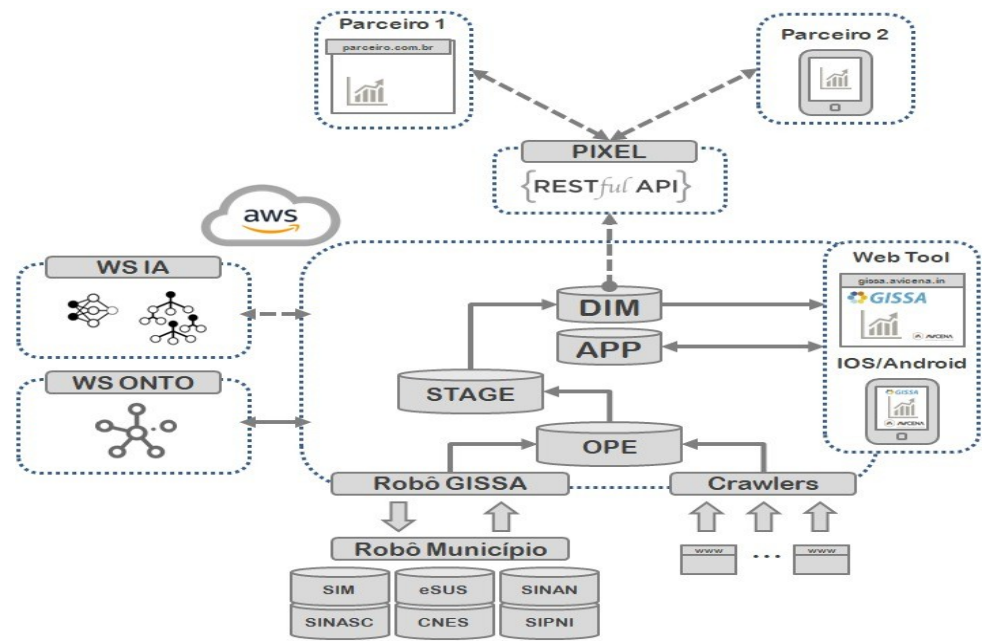

Figure 4. Arquitetura PIXEL usando a PIXEL

A PIXEL surge como uma ponte no sistema, providenciando a comunicação e fazendo com que as informações dos sistemas sejam acessíveis e que o mesmo possa consumir informações de forma prática. A Figura 5 exibe a página inicial da PIXEL. Nessa página, o usuário pode escolher a base de dados de onde se deseja capturar as informações (a base de dados robô, indicada na Figura 5). Ao selecionar a base de dados, aparecem suas tabelas (a tabela pessoa foi escolhida), que podem ser escolhidas para disponibilizar suas informações via Restful ao solicitante. Tendo escolhido a base de dados e a tabela, cria-se o modelo com as informações pré-definidas, como tipo de dados e tamanho (Figura 6). Na Figura 6, pode-se ver o modelo criado pela PIXEL na base de dados do solicitante.

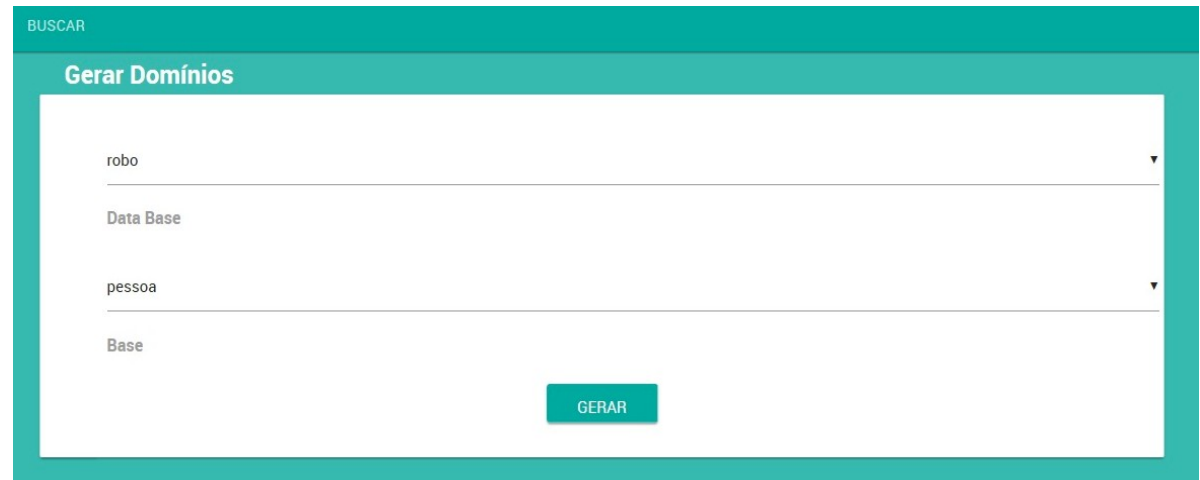

Figure 5. Base de dados e tabelas selecionadas.

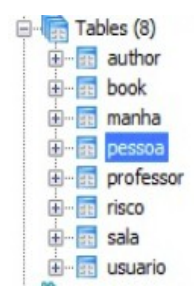

Figure 6. Criação do modelo. 


\section{Considerações Finais}

Plataformas interoperáveis desempenham papéis fundamentais para alcançar níveis de integração entre sistemas. A integração possibilitada mediante a utilização da plataforma PIXEL foi rápida, pode ser vista hoje em prática no framework GISSA, um produto que já se encontra em atividade nos estados do Ceará e Pernambuco. A abordagem de criação dinâmica de endpoints oferecida pela PIXEL permite uma evolução para estruturas complexas e com maior potencial de integração de sistemas, mesmo que estes usem tecnologias legadas. A PIXEL foi validada no framework GISSA, nada impede que essa solução possa ser adaptada para ser executada em sistemas similares e não se limita apenas à área da saúde, tendo em vista que seus componentes são modulares. Foram alcançadas as interoperabilidades: fundamental via gerador de modelos, a sintática via ApiPIXEL e a semântica via GIRLS.

Como trabalhos futuros, pretende-se realizar o mecanismo from-to, para tornar os campos dinâmicos e suscetíveis a mudanças. Será realizado o tunelamento de carga, visando trabalhar com alta quantidade de informações na PIXEL e serão criados recursos além do uso de tabelas para gerar endpoins possibilitando a utilização de banco não relacionais.

\section{Referências}

Amin, M. M., Sutrisman, A., Stiawan, D., Ermatita, E., Alzahrani, M. Y., and Budiarto,R. (2020). Interoperability framework for integrated e-health services. Bulletin of Electrical Engineering and Informatics, 9(1):354-361.

do Nascimento, S. C., Carvalho, F. O., and da Rocha, T. (2013). Um framework para interoperabilidade entre componentes distribuídos heterogêneos. Revista Brasileira de Administração Científica, 4(2):239-256.

dos Santos, E. M. (2011). A adoção da arquitetura e-ping: Um estudo de caso na Fiocruz/Bahia.

Gomes, F., Freitas, R., Ribeiro, M., Moura, C., Andrade, O., and Oliveira, M. (2019).Girls, a gateway for interoperability of electronic health record in low-cost system:*interoperability beetween fhir and openehr standards. In 2019 IEEE International Conference on E-health Networking, Application \& Services (HealthCom), pages 1-6. IEEE.

Henning, F. (2013). Adoption of interoperability standards in government information networks: an initial framework of influence factors. In Proceedings of the 7th International Conference on Theory and Practice of Electronic Governance, pages 264-267. ACM.

Hidalgo-Delgado,Y.,Xu, B., Mariño-Molerio, A. J., Febles-Rodríguez, J. P., and LeivaMederos, A. A. (2019). A linked data-based semantic interoperability framework for digital libraries. Revista Cubana de Ciências Informáticas, 13(1):14-30.

Mahmoud, K. M. (2013). A unified messaging-based architectural pattern for buildingscalable enterprise service bus. In Proceedings of International Conference on Information Integration and Web-based Applications \& Services, page 697. ACM.

Oliveira, M., Hairon, C., Andrade, O., Moura, R., Sicotte, C., Denis, J., Fernandes, S.,Gensel, J., Bringel, J., and Martin, H. (2010). A context-aware framework for healthcare governance decision-making systems: A model based on the brazilian digital tv. In 2010 IEEE International Symposium on"A World of Wireless, Mobile and Multimedia Networks"(WoWMoM), pages 1-6. IEEE.

Vernadat, F. B. (2007). Interoperable enterprise systems: Principles, concepts, and methods. Annual reviews in Control, 31(1):137-145. 